Original Research Paper

\title{
Internet-Based Teleoperation of Carts Considering Effects of Time Delay via Continuous Pole Placement
}

\author{
Theophilus Okore-Hanson and Sun Yi \\ Department of Mechanical Engineering, North Carolina A \& T State University, Greensboro, USA
}

\section{Article history}

Received:10-03-2017

Revised: 14-03-2017

Accepted: 20-03-2017

Corresponding Author:

Sun Yi

Department of Mechanical

Engineering, North Carolina A

\& T State University,

Greensboro, USA

Email: syi@ncat.edu

\begin{abstract}
A new control design for teleoperation considering time delay is presented. Unlike existing methods for communication delays, the design presented here identifies and continuously moves the rightmost eigenvalues to desired positions. Signal transmission through the Internet using protocols (e.g., UDP) introduces time delays into the system. The time delays cause instability as the system is forced to make use of past information rather than the present in determining the output one agent sends to another agent. The proposed method controls rightmost eigenvalue in infinite spectrum such that the command signal to the cart ensures a sustained stabilized system. Experimental results are presented to validate the design method.
\end{abstract}

Keywords: Communication Delays, Eigenvalues, Teleoperation, UDP, Stabilization

\section{Introduction}

This paper looks at the stabilization of teleoperated systems under time delays. Systems react to external actions and the reaction is typically not instantaneous due to transport and propagation phenomena. Including information about the systems' past in the model, can lead to instability of time delayed systems. Our aim in this study is to understand the effects of time delays on the stability of teleoperation and design a control system to reverse the adverse effects of the delay. Results are validated through experiment. For comparison, stable PV controller is designed for the teleoperation system without consideration of time delay using classical method. The gains are applied to a time delay system to investigate performance under time delay. The PV controller is then modeled considering time delay and the robustness is investigated. The design is validated with an experiment using two cart systems and application of the calculated controller gains.

Time delays are inherent in various processes both natural and manmade, in biology, medicine, chemistry, physics, engineering, economics. Hence, modeling systems without delay can be approximations of the real life models (Kuang, 1993). For example, the presence of delay in an automatic regulator system may cause the appearance of self-exciting oscillations, of increase of overregulation and even of instability of the system (Norkin, 1972). Communicating between agents over the Internet also causes time delay. A perfect controller designed without consideration of time delay may become unstable due to the effects of the time delay. Time delay turns a finite-dimensional system into an infinite-dimensional system. The controller design methodology was developed based on eigenvalue based continuous pole placement method (Michiels et al., 2002). The aim is to control the rightmost characteristic roots in a quasi-continuous way at desired locations in the Left Half-Plane (LHP). The difficulty is controlling infinite number of characteristic roots with finite number of controller parameters. In addition, nonlinearities in typical manipulators further complicate the analysis (Anderson and Spong, 1989). Two carts are subsequently used to implement the controller to experimentally validate the results.

User Datagram Protocol (UDP), one of the transport layer protocols, is used to create process to process communication path for the cart system. The two host computers communicate through their IP addresses. Time delay arises due to connectionless information transfer through the Internet. In transporting signals from the master agent to the slave agent and feedback signals from the slave to the master, time delay arises. This delay causes undesired responses and instability. 
Therefore, there is need to design a control system capable of stabilizing the plants considering the effects of the time delay. First a stable controller is designed without consideration of time delay and tested with time delay. The DDE-BIFTOOL is used to compute branches of steady-state solution. The DDE-BIFTOOL is a collection of routines written in MATLAB (Engelborghs et al., 2000) mostly used in scientific computing environments. Delay Differential Equations (DDEs) differ from Ordinary Differential Equations (ODEs) in that the derivative at any time depends on the solution at prior times. The package provides a tool for numerical bifurcation analysis of steady state solutions and periodic solutions of delay differential equations with multiple fixed, discrete delays. The DDEBIFTOOL is used to find the critical eigenvalues of the system. Given an equilibrium, it allows to approximate the rightmost, stability determining roots of the infinite dimensional characteristic equation. The results from the DDE-BIFTOOL are compare to simulation results and finally implemented on the twocarts system for validation.

The paper is organized as follows; Section 2 presents an overview of the experimental setup used for the study. In section 3 , modeling of a teleoperation system with time delay is presented. Stability analysis is presented in section 4. Simulation and experimental results are presented in 5 and lastly, conclusions from the study are presented in section 6 .

\section{Experimental Setup}

Two carts made by Quanser with two clients and Quarc UDP Server are used for the experiment. Multiagent synchronization without the effects of time delay was presented in (Okore-Hanson and Yi, 2016). This paper looks at synchronization of two cart systems under the effects of time delay.

Time delay in transferring the signal from the master agent to the slave agent can be noticed by the motion of the slave.

The setup is shown in Fig. 1. The setup consists of the following:

- Two Carts with DC motors and encoder sensors

- QPID data acquisition device by Quanser

- 2 voltage amplifiers

- 2 computers and hosts for implementing UDP communication using Matlab/Simulink

The UDP transport protocol is responsible for creating a process to process connection and ensuring that the packet data gets to the correct process. If a stream of data is produced, the transport layer takes this stream of data from the application layer and break them into packets. Since UDP does not need to retransmit lost packets it is advantageous especially in situations where delay is of concern. The data is sampled at a rate of $0.002 \mathrm{~Hz}$.

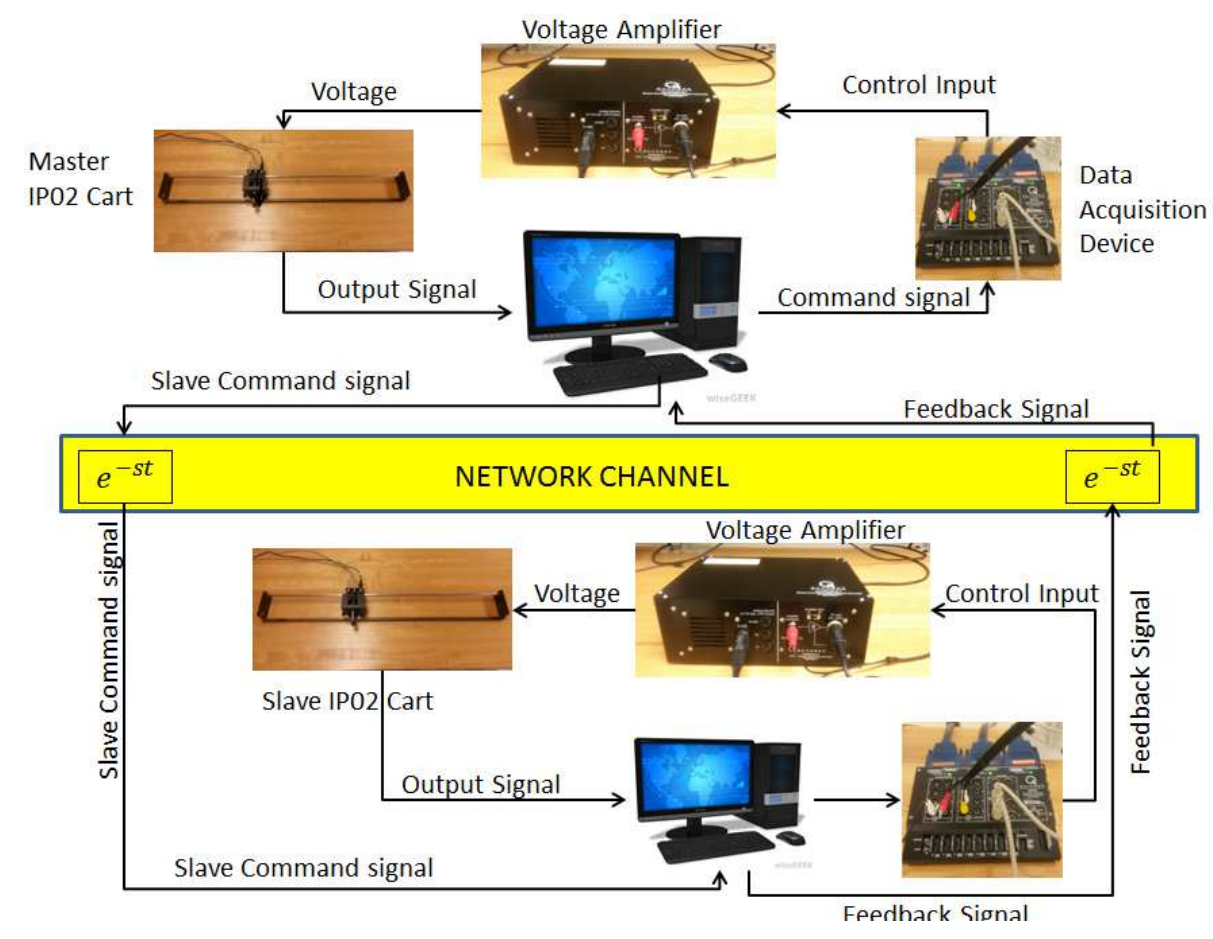

Fig. 1. Schematic diagram of experimental tele-robotic system 


\section{Modeling and Control Design}

Generalized Delay Differential Equations (DDEs) can be represented in a matrix-vector form as:

$$
\dot{x}(t)=A x(t)+A_{d} x(t-h)
$$

where, $A$ and $A_{d}$ are $n x n$ coefficient matrices and $x(t)$ is an $n x 1$ state vector in the time domain.

The cart is operated by a Faulhaber coreless DC motor. The transfer function without time delay is:

$$
P(s)=\frac{K}{s(\tau s+1)}=\frac{X(s)}{V_{m}(s)}
$$

where, $\tau=0.05838[s]$ is the motor time constant, $K=0.1436\left[\mathrm{rad} / \mathrm{Vs}^{-1}\right]$ is the motor steady state gain, $X(s)$ is the state vector in the Laplace domain and $V_{m}(s)$ is the input voltage. The proportional-velocity controller is used to yield a stable control signal to regulate the motor position. It has the following structure for motors 1 and 2 respectively:

$$
\begin{aligned}
& V_{m 1}(s)=K_{p 1}\left(x_{d}-x_{c 1}\right)+K_{u 1}\left(x_{c 2}-x_{c 1}\right)_{(t-h)}-K_{v 1} s x_{c 1} \\
& V_{m 2}(s)=K_{u 2} x_{c 1(t-h)}-K_{p 2} x_{c 2}-K_{v 2} s x_{c 2}
\end{aligned}
$$

where, $K_{u 1}$ and $K_{u 2}$ are proportional gains for motor 1 and motor 2 delayed signals respectively. $K_{p 1}$ and $K_{p 2}$ are proportional control gains and $K_{v 1}$ and $K_{v 2}$ are velocity control gains. The displacements $x_{c 1}$ and $x_{c 2}$ are the measured positions of carts 1 and 2, respectively and $x_{d}$ is the desired reference.

Finally, the Delay Differential Equation (DDE) for the PV control is written in the matrix vector-form as:

$$
\begin{aligned}
& {\left[\begin{array}{l}
\dot{x}_{1} \\
\dot{x}_{2} \\
\dot{x}_{3} \\
\dot{x}_{4}
\end{array}\right]=\left[\begin{array}{cccc}
0 & 1 & 0 & 0 \\
\frac{-K K_{p 1}}{\tau} & -\frac{\left(1+K K_{v 1}\right)}{\tau} & 0 & 0 \\
0 & 0 & 0 & 1 \\
0 & 0 & -\frac{K K_{p 2}}{\tau} & -\frac{\left(1+K K_{v 2}\right)}{\tau}
\end{array}\right]\left[\begin{array}{l}
x_{1} \\
x_{2} \\
x_{3} \\
x_{4}
\end{array}\right]} \\
& +\left[\begin{array}{cccc}
0 & 0 & 0 & 0 \\
\frac{K K_{u 2}}{\tau} & 0 & \frac{-K K_{u 2}}{\tau} & 0 \\
0 & 0 & 0 & 0 \\
\frac{K K_{u 1}}{\tau} & 0 & 0 & 0
\end{array}\right]\left[\begin{array}{c}
x_{1(t-h)} \\
x_{2(t-h)} \\
x_{3(t-h)} \\
x_{4(t-h)}
\end{array}\right]+\left[\begin{array}{c}
0 \\
\frac{K k_{p}}{\tau} \\
0 \\
0
\end{array}\right] x_{d}
\end{aligned}
$$

where, $\left[\begin{array}{llll}x_{1} & x_{2} & x_{3} & x_{4}\end{array}\right]^{T}=\left[\begin{array}{llll}x_{c 1} & \dot{x}_{c 1} & x_{c 2} & \dot{x}_{c 2}\end{array}\right]^{T} . \quad$ The DDE-BIFTOOL is used to compute the rightmost, stability determining roots of the characteristic equation. Results from DDE-BIFTOOL and closed-loop control simulation is presented in subsequent sections. The block diagram for the PV control is shown in Fig. 2.

\section{Stability Analysis using Eigenvalues}

Stability criteria used to determine asymptotic stability of the teleoperation system is presented. The theoretical explanation is followed by simulation and experimental results using the two-cart system. Due to the infinite spectrum, it is difficult to determine the rightmost eigenvalues. The DDE-BIFTOOL is used to determine the stability of the system by allowing to calculate the rightmost, stability determining root of the characteristic Equation 4.

The general DDE system is given by

$$
\begin{aligned}
& \frac{d}{d t} x(t)=f\left(x(t), x\left(t-h_{1}\right),\right. \\
& x\left(t-h_{2}\right), \ldots \ldots, \\
& \left.x\left(t-h_{m}\right), \eta\right)
\end{aligned}
$$

where, $\quad x(t) \in \mathbb{R}^{n}, f: \mathbb{R}^{n(m+1)} * \mathbb{R}^{p} \rightarrow R^{n} \quad$ is a nonlinear smooth function depending on a number of parameters $\eta \in R^{p}$ and delay $h_{i}>0, i=1, \ldots, m$.

Derivative $\dot{x}(t)$ depends on the solution at previous time(s), it is therefore necessary to provide an initial history function to specify the value of the solution before time $t=0$.

The linearization of (5) around a solution $x^{*}(t)$ is the variational equation, given by,

$$
\frac{d}{d t} y(t)=A_{0}(t) y(t)+\sum_{i=1}^{m} A_{i}(t) y\left(t-h_{i}\right)
$$

where, using $\mathrm{f} \equiv f\left(x_{0}, x_{1}, \ldots, x_{m}, \eta\right)$ :

$$
A_{i}(t)=\left.\frac{\partial f}{\partial x^{i}}\right|_{x^{*}(t), x^{*}\left(t-h_{1}\right), \ldots, x^{*}\left(t-h_{m, n)}\right.}, i=0,1, \ldots, m
$$

If $x^{*}(t)$ corresponds to steady state solution:

$$
x^{*}(t)=x^{*} \in \mathbb{R}^{n}, \text { with } f\left(x^{*}, x^{*}, \ldots, \eta\right)=0
$$

Then the matrices $A_{i}(t)$ are constants $A_{i}(t) \equiv A_{i}$ and the corresponding variational Equation 6 leads to a characteristic equation. Define the $n \times n$ - dimensional matrix $\Delta$ as:

$$
\Delta(\lambda)=\lambda I-A_{0}-\sum_{i=1}^{m} A_{i} e^{-\lambda h i}
$$




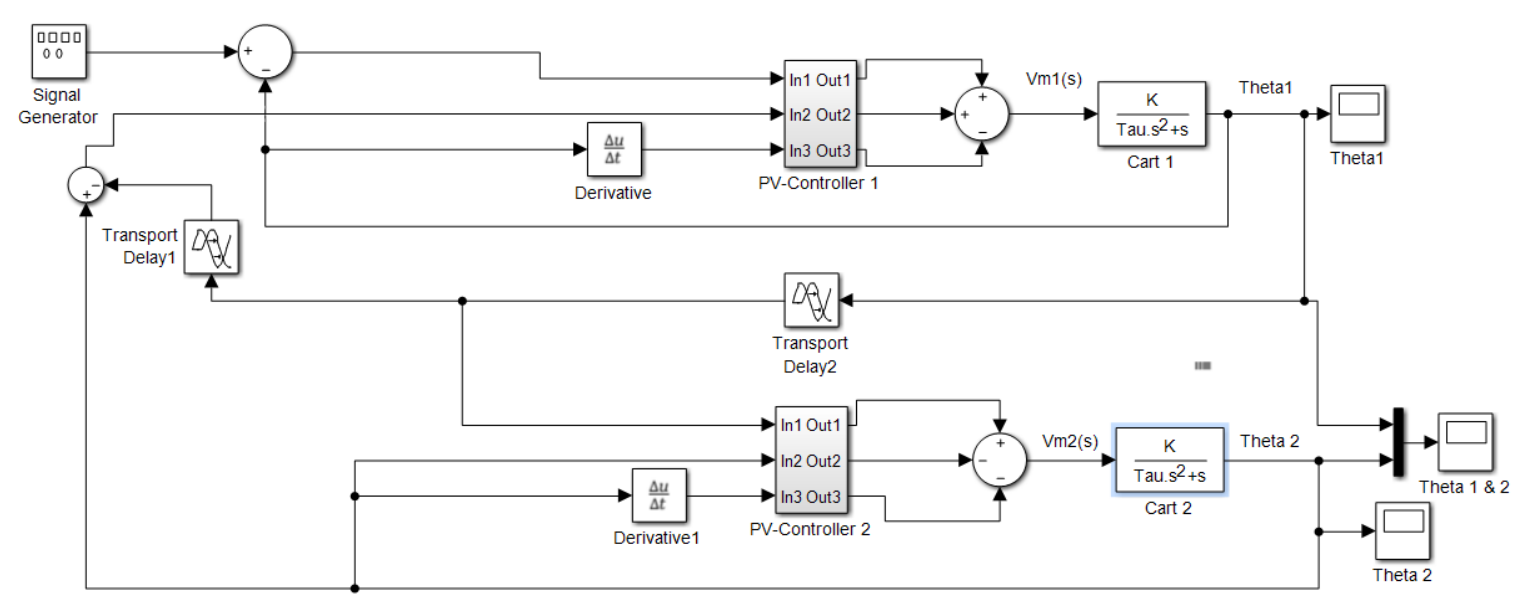

Fig. 2. Block diagram for PV control of telerobotic system

Then the characteristic equation reads:

$\operatorname{det}(\Delta(\lambda I))=0$

Equation 8 has an infinite number of roots $\lambda \in \mathbb{C}$, which determine the stability of the steady-state solution $x^{*}$. The steady state solution is (asymptotically) stable provided all roots of the characteristic equation in (8) have negative real parts (Engelborghs et al., 2000).

From (Forde and Nelson, 2004) the transcendental equation of delay differential equation, at steady state determined for $h=0$, will have the form:

$$
P(\lambda, h) \equiv P_{1}(\lambda)+P_{2}(\lambda) e^{-\lambda h}=0
$$

where, $h$ is the length of discrete time delay and $P_{1}$ and $P_{2}$ are polynomials in $\lambda$. We can rewrite Equation 9 as:

$$
\sum_{j=0}^{N} a_{j} \lambda^{j}+e^{-\lambda \tau} \sum_{j=0}^{M} b_{j} \lambda^{j}=0
$$

and assume that the steady state about which we have linearized is stable in the absence of delay. Therefore, for $h=0$ all the roots of the polynomial have negative real part. As $h$ varies, the roots change. We are interested in any critical value of $h$, such that the characteristic equation has a purely imaginary root (Norkin, 1972). That is to determine whether bifurcation can occur as a result of the introduction of delay.

After finding a critical delay $h^{*}$ and the point $\lambda=i v^{*}$ at which the roots of a characteristic equation hit the imaginary axis, it is necessary to confirm that the root continues into the positive half-plane as $h$ increases past $h^{*}$.

\section{Simulation and Experiment}

The cart is operated by a DC motor with the following characteristics: Time constant $\tau=0.05838[\mathrm{~s}]$ and the motor steady-state gain $K=0.1436\left[\mathrm{rad} / \mathrm{Vs}^{-1}\right]$. To estimate the time delay in the UDP communication setup through the internet, the responses of the master and slave were plotted on the same axis and the time delay was estimated from the difference between the two responses. The time delay for the UDP cart system was estimated to be $h=1.62[\mathrm{secs}]$.

Figure 3 shows the response from gains calculated for a time delay $h=0$ in Table 1 . Without time delay the above calculated gains yield a stable response as shown in Fig. 3, however with time delay the system becomes unstable as shown in Fig. 4. It is therefore important to consider time delay when designing systems with delayed signals.

The controller gains calculated at $h=0$ were applied to Simulink block diagram with time delay $h=$ 1.62[secs], the unstable output is presented in Fig. 4.

Using the DDEBIFTOOL with a time delay $h=$ $1.62[\mathrm{secs}]$ and the parameters in Table 1 obtained at $h$ $=0$, instability of the system with the rightmost eigenvalue $\mathfrak{R}(\lambda)=0.063$ is confirmed. Result of the DDEBIFTOOL is shown in Fig. 5. The stable eigenvalues calculated without considering delay are shown in blue circles. The result agrees with the earlier results obtained in Fig. 4. Time delay shifts the rightmost eigenvalues to the unstable region.

In order to verify the obtained results, the parameters calculated at time delay $h=0$ are applied to the Carts system and the result is displayed in Fig. 6.

Figure 7 shows poles obtained with the DDEBIFTOOL from gains calculated at $h=1.62$ [secs]. The obtained results prove that time delay can limit and degrade the achievable performance of controlled systems and even induce instability (Yi et al., 2010). It is therefore imperative to consider time delay when designing control systems. 


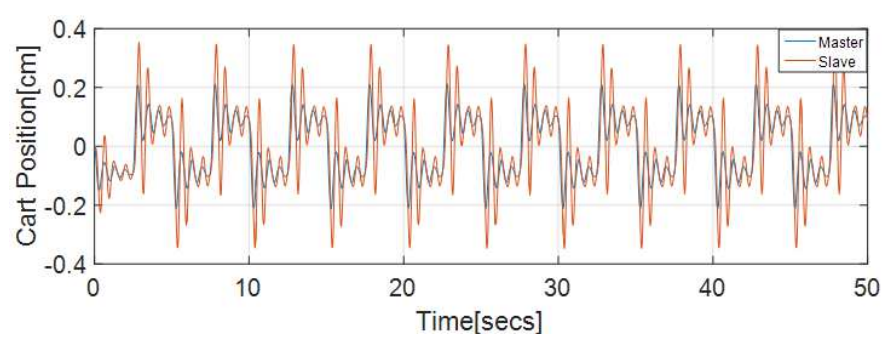

Fig. 3. PV Control simulated cart response at delay $h=0$ and $\Re(\lambda)=-1.1853$

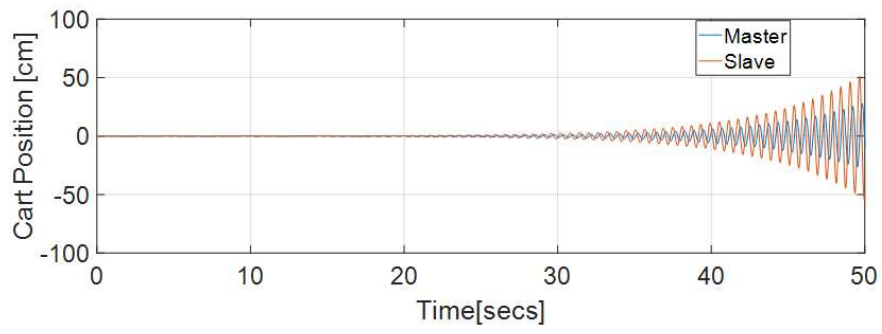

Fig. 4. PV control simulated response at time delay $h=1.62[$ secs $]$

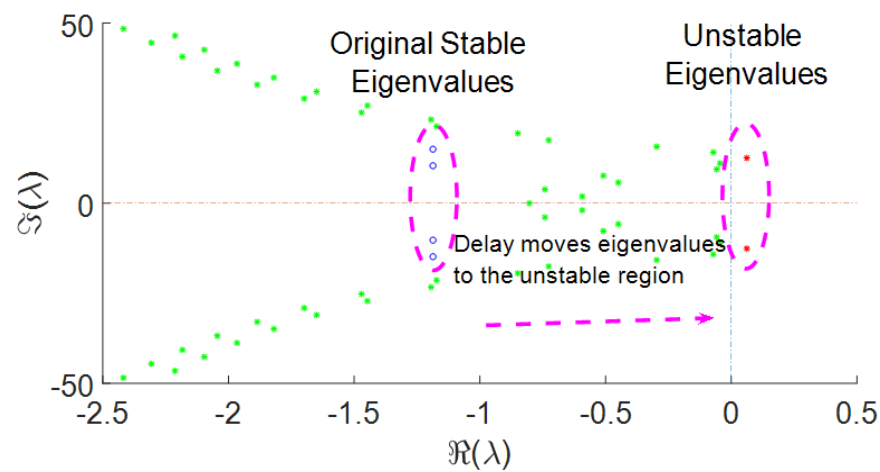

Fig. 5. Rightmost eigenvalue $\mathfrak{R}(\lambda)=0.138$ for delay $T_{d}=1.62[\operatorname{secs}]$

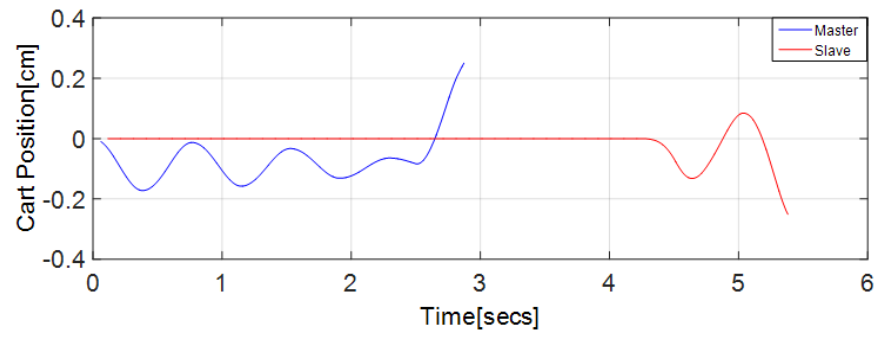

Fig. 6. Cart position from actual system with control parameters calculated at delay $h=0$

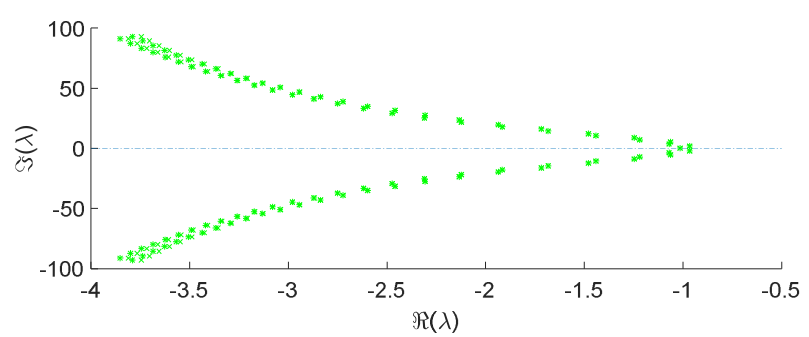

Fig. 7. Rightmost eigenvalue $\mathfrak{R}(\lambda)=-0.965$ delay $h=1.62[\mathrm{secs}]$ 


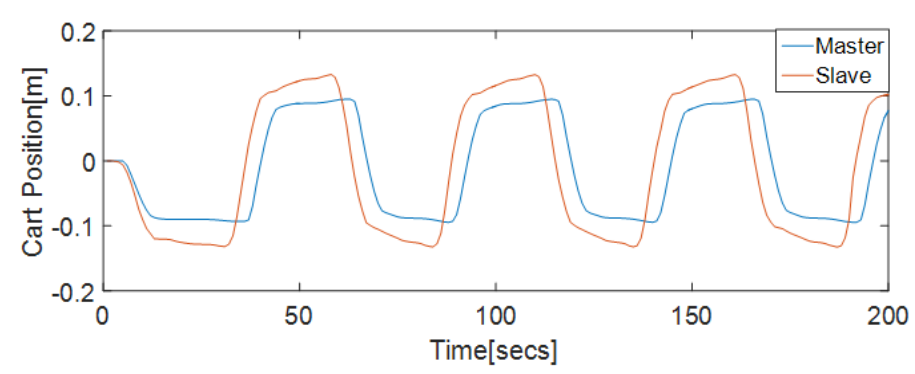

Fig. 8. Simulated cart response time delay $h=1.62[\mathrm{secs}]$ rightmost eigenvalue $\mathfrak{R}(\lambda)=-0.965$

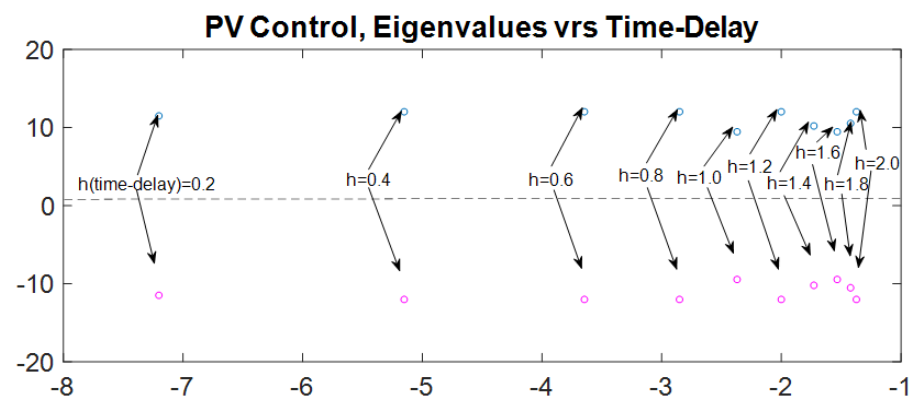

Fig. 9. Continuous shift of rightmost eigenvalue as time-delay changes

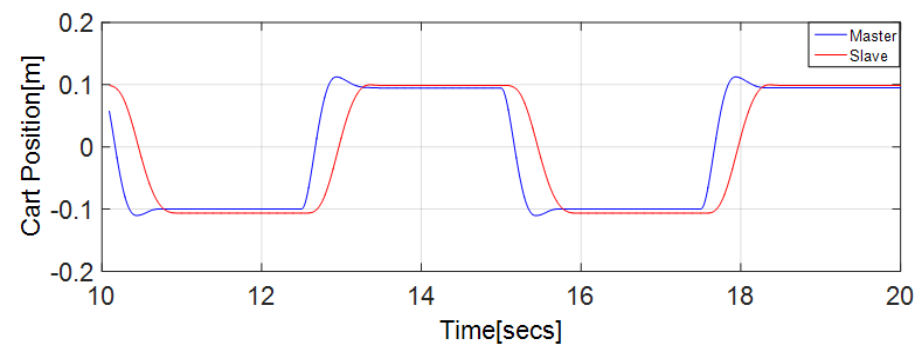

Fig. 10. Experimental cart response PV-controller

Table 1. Controller gains calculated with time delay, $\mathrm{h}=0$

\begin{tabular}{ll}
\hline$K_{p}$ & $K_{u}$ \\
\hline$K_{p 1}=50, K_{p 2}=80$ & $K_{u 1}=5, K_{u 2}=80.0$ \\
\hline
\end{tabular}

Table 2. Controller gains with delay $\mathrm{h}=1.62 \mathrm{~s}$ and $\mathfrak{R}(\lambda)=-0.965$

\begin{tabular}{ll}
\hline$K_{p}$ & $K_{v}$ \\
\hline$K_{p 1}=50, K_{p 2}=35$ & $K_{v 1}=K_{v 2}=0.5$ \\
\hline
\end{tabular}

Table 3. Controller gains for $\mathrm{h}=1.62 \mathrm{~s}$ and $\lambda=-1.12$

\begin{tabular}{ll}
\hline$K_{p}$ & $K_{v}$ \\
\hline$K_{p 1}=80, K_{p 2}=35$ & $K_{v 1}=K_{v 2}=0.5$ \\
\hline
\end{tabular}

In the continuous pole placement method used in this study, unstable eigenvalues are shifted to the stable region by adjusting the sensitivity of the rightmost eigenvalues with respect to the feedback gains. The rightmost eigenvalues are first computed using the DDEBIFTOOL. The computed eigenvalues are moved in the direction of the left half-plane by applying small changes to the feedback gains using their sensitivities.
Response from a PV controller with controller gains in Table 2 obtained by sensitivity analysis explained above considering time delay $h=1.62[\mathrm{secs}]$ are shown.

Simulation of the response with the controller gains obtained by the continuous pole placement method is shown in Fig. 8 with stabilized output.

Using the continuous pole placement method, the unstable eigenvalues can be shifted to the left half plane in a quasi-continuous way by applying small changes to the feedback gain, while monitoring the eigenvalues with large real parts (Michiels et al., 2002). Figure 9 shows continuous variation of the eigenvalues with change in time delay.

The continuous pole placement is used to obtain the controller gains in Table 3. The controller gains are applied to the cart system through the internet and the response is displayed in Fig. 10.

\section{Conclusion}

A stabilization control method in teleoperation was developed and implemented. The paper showed that a 
stabilized control system without consideration of the effect of time delay may yield instability. The designed controller considering the effect of time delay effectively stabilized the teleoperated system using the Cart systems. The DDE-BIFTOOL in Matlab provided an efficient tool in determining the rightmost eigenvalues. This method is very efficient especially when the dimension of the delay differential equation system is large.

\section{Author's Contributions}

All authors contributed to the writing of the manuscript.

\section{Ethics}

No part of this article may be reproduced without written permission from the publisher or authors.

\section{References}

Anderson, R.J. and M.W. Spong, 1989. Bilateral control of teleoperators with time delay. IEEE Trans. Automatic Control, 34: 494-501. DOI: $10.1109 / 9.24201$

Engelborghs, K., T. Luzyanina and G. Samaey, 2000. DDE-BIFTOOL: A Matlab package for bifurcation analysis of delay differential equations. TW Report.
Forde, J. and P. Nelson, 2004. Applications of Sturm sequences to bifurcation analysis of delay differential equation models. J. Math. Anal. Applic., 300: 273-284. DOI: 10.1016/j.jmaa.2004.02.063

Kuang, Y., 1993. Delay Differential Equations: With Applications in Population Dynamics. 1st Edn., Academic Press, Boston, ISBN-10: 0080960022, pp: 398.

Michiels, W., K. Engelborghs, P. Vansevenant and D. Roose, 2002. Continuous pole placement for delay equations. Automatica, 38: 747-761. DOI: $10.1016 / \mathrm{S} 0005-1098(01) 00257-6$

Norkin, S., 1972. Introduction to the theory and Application of Differential Equations with Deviating Arguments. 1st Edn., Academic Press.

Okore-Hanson, T. and S. Yi, 2016. Adaptive synchronization of networked multi-agent systems considering transient responses and disturbances. Proceedings of the International Symposium on Flexible Automation, Aug. 1-3, IEEE Xplore Press, pp: 490-496. DOI: 10.1109/ISFA.2016.7790211

Yi, S., P.W. Nelson and A.G. Ulsoy, 2010. Time-Delay Systems: Analysis and Control using the Lambert W Function. 1st Edn., World Scientific, ISBN-10: 9814307408, pp: 156. 